\section{Lumbosacral plexopathy from iliopsoas haematoma after combined general- epidural anaesthesia for abdominal aneurysmectomy}

\author{
Edward T. Crosby MD FRCPC, * \\ Dennis R. Reid MB CHB FRCPC FRCA(Eng), * \\ Gina DiPrimio $M D,^{\dagger}$ \\ Steven Grahovac MD FRCPC ${ }^{\dagger}$
}

Purpose: To report a case of iliopsoas haematoma after resection of an abdominal aortic aneurysm which resulted in a lumbosacral plexopathy.

Clinical features: An 81-yr-old man presented with an abdominal aortic aneurysm for aneurysmectomy and tube grafting. An epidural catheter was placed at the $L_{1-2}$ spinal level and combined epidural-general anaesthesia was provided for surgery. The surgery was complex and a suprarenal clamp was necessary to obtain proximal control. A continuous infusion of demerol through the epidural catheter was prescribed for postoperative analgesia. On the first postoperative day, examination revealed a paretic, pulseless right leg and he was returned to the operating room for femoral-femoral bypass. By the following day, the motor and sensory impairment had progressed to complete paralysis with loss of all deep tendon reflexes and absent sensation below $\mathrm{L}_{1}$, despite palpable pulses in the leg. A CT of the abdomen demonstrated a right iliopsoas haematoma. There was no evidence of either disc herniation or an epidural haematoma. A diagnosis of lumbosacral plexopathy secondary to a iliopsoas haematoma was made.

Conclusion: lliopsoas haematoma is a rare cause of postoperative neurological deficit following aortic vascular surgery. The haematoma results in compression of the lumbosacral neural elements and typically presents as a femoral neuropathy. The diagnosis is clinical and can be readily validated with computed tomography.

Objectif : Rapporter un hématome du psoas-iliaque compliqué d'une plexopathie lombosacrée survenue après la résection d'un anévrisme de l'aorte abdominale.

Éléments cliniques : II s'agit d'un homme de 81 ans affligé d'un anévrisme de l'aorte abdominale programmé pour une résection d'anévrisme avec greffe. Une association anesthésie épidurale-générale a été administrée avec l'installation d'un cathéter épidural à $\mathrm{L}_{1-2}$. La chirurgie était complexe et on a dû utiliser un clamp suprarénal pour le contrôle proximal. L'analgésie postopératoire était assurée par une perfusion péridurale continue de démérol. Le lendemain de l'intervention, l'examen révélait une parésie avec absence de pouls au membre inférieur droit nécessitant une réintervention pour dérivation fémoro-fémorale. Le jour suivant, l'atteinte motrice et sensorielle droite évoluait vers la paralysie complète avec perte de tous les réflexes tendineux profonds et absence de sensation sous le niveau $L_{1}$ malgré la présence de pouls palpables à la jambe. Une tomographie axiale de l'abdomen démontrait un hématome du psoas-iliaque droit. On n'a pu mettre en évidence de hernie discale ou d'hématome épidural. On portait le diagnostic de plexopathie lombosacrée secondaire à un hématome du psoas-iliaque.

Conclusion : L'hématome du psoas-liliaque constitue une cause rare de déficit neurologique après une chirurgie vasculaire majeure. L'hématome provoque une compression des éléments nerveux lombosacrés et se présente typiquement comme un neuropathie fémorale. Ce diagnostic clinique peut être validé rapidement par tomographie axiale.

From the Departments of Anaesthesia ${ }^{\star}$ and Radiology, ${ }^{\dagger}$ University of Ottawa, Ottawa General Hospital. Address correspondence to: Dr. Edward Crosby, Department of Anaesthesia, Room 2600, 501 Smyth Road, Ottawa, Ontario K1H 8L6; Phone: 613-737-8187; Fax: 613-737-8189; E-mail: ecrosby@fox.nstn.ca. Accepted for publication October 3, 1997. 


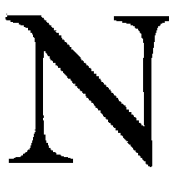

EW onset neurological findings after anaesthesia and surgery warrant thorough evaluation to determine both the aetiology and the potential for resolution with intervention. The diagnosis may be delayed when regional blocks are utilised to provide postoperative analgesia as the neurological deficit may be attributed to the block. Persistent neuraxial block, especially those that are not anatomically characteristic (i.e., a unilateral block), should prompt neurovascular assessment to rule out more serious aetiology before the deficit is presumed to be a prolonged drug effect. This case report describes a patient with an unusual cause of postoperative neurological deficit transiently attributed to an epidural block.

\section{Case report}

An 81-yr-old man presented with a $6 \mathrm{~cm}$ infra-renal abdominal aortic aneurysm for ancurysmectomy and tube grafting. The aneurysm was obstructing the right ureter, resulting in hydronephrosis. He had a history of chronic obstructive lung disease, previous myocardial infarction and congestive cardiac failure. He was admitted to the intensive care unit (ICU) the day before surgery for placement of a pulmonary artery catheter and cardiorespiratory optimisation. The following day, he was taken to the operating room for planned surgery. An epidural catheter was placed at the $\mathrm{L}_{1-2}$ spinal level, via a midline approach, using a loss of resistance to air technique, without incident. The catheter was threaded $5 \mathrm{~cm}$ into the epidural space and activated with $11 \mathrm{ml}$ bupivacaine $0.5 \%$ and $50 \mathrm{mg}$ demerol after a test dose of $60 \mathrm{mg}$ lidocaine with $15 \mu \mathrm{g}$ of epinephrine. General anaesthesia was induced with $30 \mu \mathrm{g}$ sufentanil and 125 $\mathrm{mg}$ thiopentone and tracheal intubation was facilitated with $50 \mathrm{mg}$ rocuronium. Anaesthesia was maintained with isoflurane $0.2-0.4 \%$ (endtidal concentration) in nitrous oxide $50 \%$ in oxygen and an infusion of $0.002-0.004 \mu \mathrm{gg} \cdot \mathrm{kg}^{-1} \cdot \mathrm{min}^{-1}$ sufentanil; muscle relaxation was maintained with incremental 1-2 mg doxacurium. The surgery was complex. The aneurysm was inflammatory and a suprarenal clamp was necessary to obtain proximal control. Distal control could not be obtained by clamp at the iliac level and Fogarty catheters were used to control back-bleeding. Surgery lasted for four hours and blood loss was estimated at five litres. $\mathrm{He}$ received six litres crystalloid, one litre pentastarch, 2.61 washed, salvaged blood and four units allogeneic blood. Following surgery, he was returned to the ICU in satisfactory condition with a blood pressure of 104/50 $\mathrm{mmHg}$, a heart rate of $74 \mathrm{bpm}$ and oxygen saturation of $100 \%$. A continuous infusion of demerol through the epidural catheter was prescribed for postoperative anal- gesia. His early postoperative course was complicated by renal failure with fluid overload and interim dialysis was required. He was discharged to the ward on the seventh day after operation. His further course was complicated by $E$. coli sepsis, and pneumonia with recurrent pleural effusion. He spent three months in hospital before discharge.

Overnight following the surgery, his right leg was noted to be mottled in appearance and cool to touch, with absent pulses and obvious motor impairment. The epidural infusion was discontinued and the leg reexamined, revealing a paretic and pulseless leg. A diagnosis of ischaemic neuropathy was made and he was returned to the operating room for femoral-femoral bypass on the morning of the first postoperative day. Following surgery, it was noted that, although peripheral pulses were now established, he had minimal hip and knee flexion, absent patellar reflexes and near complete sensory blockade. The infusion of epidural demerol was again stopped, the catheter removed and urgent computerised tomography was scheduled. A CT of the abdomen (Figure 1), demonstrated a right iliopsoas haematoma; there was no evidence of either disc herniation or a central epidural haematoma. A diagnosis of lumbosacral plexopathy secondary to an iliopsoas haematoma was made.

An electromyogram was abnormal, demonstrating denervation in all of the muscles of the right leg consistent with the clinical diagnosis of a lumbosacral plexopathy. No sensory or motor responses were obtained on the nerve conduction studies, implying

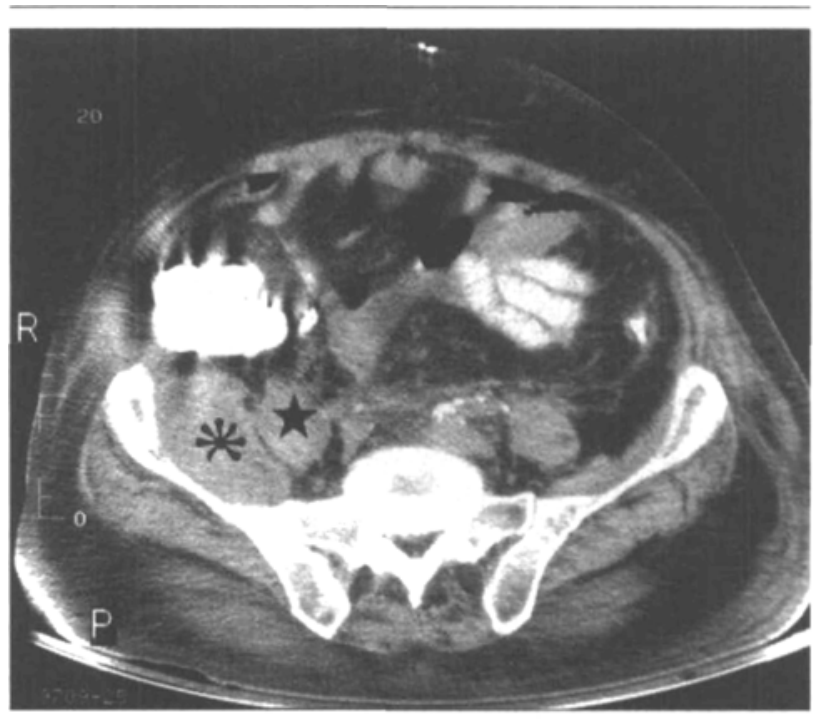

FIGURE 1 Abdominal CT scan. On the right side, both an iliacus (star) and psoas haematoma (asterisk) are evident. 
severe axonal degeneration in these nerves and a poor prognosis for recovery. An incidental note was made of an underlying sensory motor axonal neuropathy on the basis of left-sided studies which was, perhaps, related to the patient's critical illness or may have predated his admission.

By the second postoperative day his condition had progressed to complete paralysis of the right leg with loss of all deep tendon reflexes and absent sensation below the level of $L_{1}$. At the time of discharge, three months postoperatively, he had little improvement in function and had developed a neuropathic pain syndrome affecting the leg.

\section{Discussion}

We report a case of lumbosacral plexopathy resulting from haematoma formation following a complex aortic aneurysm resection. The haematoma involved the iliopsoas muscle and presumably compressed the elements of the lumbosacral plexus at that level. This is an uncommon association after aortic vascular surgery. Imaging is not done routinely after abdominal aortic grafting and there is no information of the best means of managing patients who develop iliopsoas haematoma following aortic reconstruction or how often these are symptomatic. Clearly, with any acute postoperative neurological deficit, early diagnosis and differentiation of the underlying aetiology is necessary to direct interventions.

The differential diagnosis of postoperative leg weakness after abdominal aortic surgery can be divided into central lesions including those of the brain and cord (upper motor neuron) and peripheral lesions involving the roots, trunks and peripheral nerves (lower motor neuron). Possible central lesions in this case include an intracranial ischaemic lesion, a spinal cord ischaemic injury as a result of the aortic cross-clamp, a haematoma of the central canal related to the epidural catheter placement and concurrent anticoagulation, or a persistent effect of the epidural block. ${ }^{1-3}$ Peripheral lesions include lumbosacral plexopathy (root and trunk lesions) or an ischaemic neuropathy. Ischaemic neuropathy was the most likely alternative diagnosis in this case. Finally, neuropathy is increasingly recognised as a feature of critical illness (i.e., critical illness polyneuropathy and mononeuritis multiplex) although it typically presents as generalised rather than focal weakness. ${ }^{4}$

Three characteristics were cited by the neurological consultant to support the diagnosis of a compressive rather than an ischaemic neuropathy. First, was the absence of ischaemic leg pain during the entire course, even after the epidural effect had abated. Second, was the progression of symptoms despite re-establishment of circulation after the second grafting procedure.
Third, was the extreme nature of the motor impairment, affecting the leg from the hip down with complete paralysis.

Although the epidural catheter and block were transiently implicated as aetiological factors ("guilt by association") the unilateral nature of the palsy focused attention on peripheral (lower motor neuron) causes. Both the clinical evaluation and the confirming laboratory assessments were consistent with the diagnosis of lumbosacral plexopathy, the CT scan provided the aetiological basis for the complication. In patients who have an epidural catheter in place at the time of diagnosis of new onset neurological deficits, there may be some value in leaving it in situ during the imaging process rather than removing it as was done in our case. The area of neuraxis around the catheter can be assessed for proximate abnormalities to determine if the catheter is a suspect for causation.

Veselis reported a case in which there was a delayed diagnosis of a perioperative occlusion of an iliac artery resulting in a femoral neuropathy as the finding was initially attributed to persistence of epidural bupivacaine injected to provide perioperative anaesthesia and analgesia for cystectomy. ${ }^{5}$ In patients at risk for perioperative neurovascular compromise, the diagnosis of "persistent local anaesthetic effect" to explain a new onset neurological deficit should be a diagnosis of exclusion, made after a negative evaluation for other aetiological factors. In addition, the routine use of dilute solutions of opioid and local anaesthetic agents for postoperative epidural analgesia may increase the likelihood of earlier diagnosis of new onset neurological deficits attributable to other serious aetiological factors.

\section{Relevant anatomy and pathopbysiology}

The iliopsoas compartment consists of all of the muscles covered by the iliopsoas fascia including the greater psoas, smaller psoas and iliacus muscles. ${ }^{6}$ They function as the primary flexors of the thigh and trunk. The iliacus muscle arises from the iliac wing and inserts into the psoas tendon and the lesser trochanter of the femur. The greater psoas muscle originates from the transverse processes of $T_{12}$ and the lumbar vertebrae and extends inferiorly to merge with the iliacus muscle at the $\mathrm{L}_{1}-\mathrm{S}_{2}$ levels, becoming the iliopsoas muscle. The iliopsoas muscle passes beneath the inguinal ligament to insert on the lesser trochanter of the femur via the psoas tendon. The smaller psoas muscle is a long, slender muscle located anterior to the greater psoas muscle. It arises from the sides of $T_{12}$ and $L_{1}$ and the fibrocartilage between them. Inferiorly, this muscle ends in the long, flat tendon that inserts into the iliopectineal eminence of the innominate bone. 
The femoral nerve is formed in the depths of the psoas muscle from the second, third, and fourth lumbar nerve-roots. It descends obliquely through the muscle fibres to emerge at its lateral border, a short distance above the inguinal ligament (Figure 2). The nerve then passes over the pelvic brim and under the inguinal ligament in the groove between the psoas and iliacus muscles, deep to the fascia iliaca, and enters the femoral triangle, finally dividing into its terminal branches. The femoral nerve gives motor innervation to the iliacus, pectineus, sartorius, and quadriceps femoris muscles. It supplies sensory innervation to the skin of the distal two-thirds of the anteromedial aspect of the thigh and the medial aspect of the leg.

The femoral nerve together with the psoas and iliacus muscles are situated within a very tight fascial envelope and may be compressed by a haematoma. In haemophiliacs and during the course of heparin therapy, femoral neuropathy appears to be due to a spontaneous haemorrhage into the iliacus muscle. ${ }^{7-9}$ The femoral nerve is also subject to compression neuropathy secondary to compartment syndrome resulting from expansion of either the iliacus or psoas muscles because it traverses the intermuscular groove and is wrapped in the intrinsic fascia of both muscles. Although case reports do not give a reliable indication of the duration of symptoms before recognition of pathology and appropriate treatment, it is likely that earlier detection allows a more favourable outcome by decreasing duration of nerve compression.

The blood supply of the intrapelvic portion of the femoral nerve is derived from the iliolumbar artery, a branch of the internal iliac artery, and from the deep circumflex iliac artery, a branch of the external iliac artery. On the right side, the deep circumflex artery gives more branches to the nerve and there are more anastomoses with the fourth and fifth lumbar arteries than on the left. The blood supply to the femoral nerve in the iliopsoas gutter is not as rich as that proximal or distal to the gutter, making the femoral nerve particularly susceptible to ischaemia while in the gutter. ${ }^{10}$

Boontje and Haaxma reported a $3.4 \%$ incidence of postoperative femoral neuropathy in 1006 patients having aortic surgery for aorto-occlusive or aneurysmal disease. ${ }^{11}$ The patients presented with paresis of the quadriceps muscle, hypoaesthesia of the anterior and medial thigh and lower leg and diminution or absence of the patellar tendon reflex. The syndrome was most commonly unilateral (30/34 patients, $88 \%$ ) and typically left sided (23/34 patients, $68 \%$ ). They suggested that the aetiology was ischaemic injury and resulted from disruption of blood flow to the nerve during aortic cross-clamping. They suggested that the

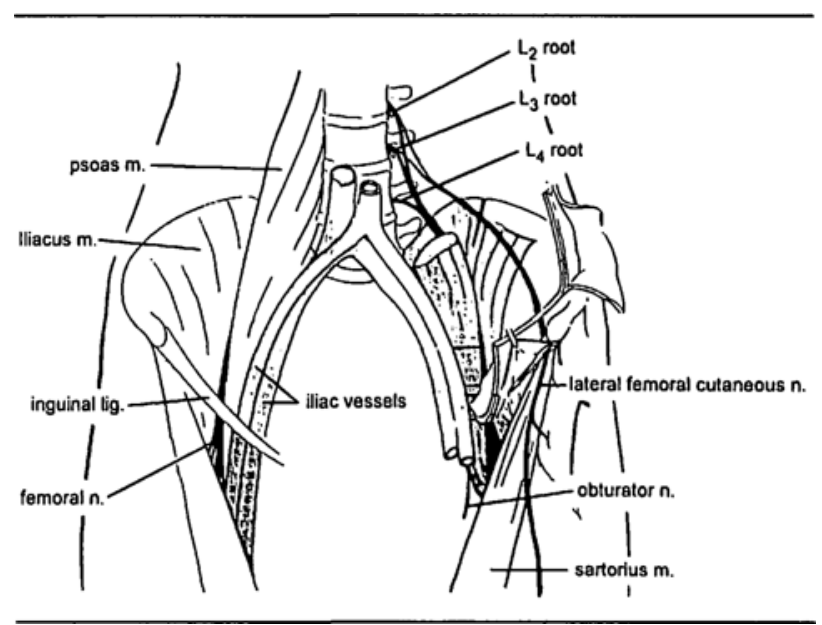

FIGURE 2 Anatomy of the iliopsoas compartment and the lumbosacral elements.

right-sided nerve was preferentially spared because of a higher degree of collateral supply. However, they ruled out iliopsoas haematoma as a cause of the neuropathy solely on the basis of clinical assessment.

The obturator nerve arises from the anterior divisions of $L_{2-4}$, which form a common trunk within the substance of the psoas muscle. It also descends obliquely through the muscle fibres but, unlike the femoral nerve, it passes out of the psoas on its medial border where it pierces the fascial envelope. The nerve passes downward with the obturator vessels and leaves the pelvis through the obturator canal to enter the thigh. It provides motor efferents to the obturator, gracilis and adductor muscles and sensory innervation to the medial thigh. Haematoma of the psoas muscle could cause a combined femoral and obturator nerve palsy. The latter would manifest itself through an adductor paralysis and impaired sensation in the skin of the medial aspect of the proximal half of the thigh.

Goodfellow injected water into the iliacus and psoas muscles of fresh cadavers and noted that the psoas fascia was thin and easily distensible, and able to contain large volumes of fluid at low pressures. ${ }^{7}$ Conversely, the iliacus fascia was thick and rigidly attached. Fluid injected into the iliacus muscle produced a high pressure swelling of the iliacus muscle, which compressed the femoral nerve against the taut psoas tendon. Further injected fluid overflowed through a communication into the psoas sheath thereby surrounding the femoral nerve and compressing the obturator nerve, which lies along the medial border of the psoas muscle. Haematomas within the iliacus muscle may manifest neurological sequelae earlier than those in the psoas muscle because the iliacus is barely distensible, leading to early 
neural barotrauma. Complete femoral nerve palsy and partial obturator nerve dysfunction with good recovery of obturator nerve function but only partial recovery of femoral nerve function has been described. The disparity can be explained anatomically as fluid produces a higher pressure in the thick-walled iliacus compartment compressing the femoral nerve compared to low pressure in the thin-walled psoas compartment, compressing the obturator nerve. Also, extension of the fluid into the psoas sheath surrounded the femoral nerve produces both medial and lateral pressure on the femoral nerve.

The sciatic nerve originates from the anterior divisions of $\mathrm{L}_{4}-\mathrm{S}_{3}$. The nerve trunks fuse and leave the pelvis through the greater sciatic notch below the piriform muscle. The $\mathrm{L}_{4}-\mathrm{S}_{2}$ nerve trunks lie directly posterior to the common iliac arteries and may be compressed by an aneurysm of this artery or of its branches.

\section{Clinical presentations}

Intramuscular haemorrhage with resultant compressive neuropathy occurs in many settings although reports of spontaneous development of iliacus or psoas muscle hematoma following anti-coagulant therapy predominate in the literature. Spontaneous haemorrhage into the substance of the iliacus and psoas muscles are common haemorrhagic complications of both haemophilia and anticoagulant therapy and account for most reported cases of lumbosacral plexopathy from haematoma. Fifty-one cases of spontaneous iliacus, psoas or iliopsoas muscle haematoma related to anticoagulant therapy with oral warfarin or intravenous heparin were identified by Sasson after a MEDLINE search of the English language literature covering January 1966 to February 1995. ${ }^{12}$ All reported cases presented as the neurological sequelae of femoral nerve compression, causing quadriceps paralysis, altered sensation to the thigh and diminished patellar reflex. In Sasson's review, 26 patients had either total or near total recovery, 18 had partial recovery, four had minimal or no recovery and one patient died. No information was available for two patients.

Reports of iliopsoas haematoma and neuropathy have also been reported secondary to vascular pathology but they are far fewer than those resulting from the haemorrhagic complications of anticoagulant therapy. Razzuk reported two patients who developed femoral neuropathy secondary to ruptured aortic aneurysm. ${ }^{13}$ In both cases the rupture was contained posteriorly but resulted in iliopsoas haematoma and femoral nerve compression. Kubacz reported combined femoral, obturator and sciatic nerve palsy resulting from compression of the lumbosacral plexus by an iliopsoas haematoma after a postero-lateral rupture of an abdominal aortic aneurysm. ${ }^{14}$ The mechanism of the femoral and obturator palsy was likely the same as in our patient although the sciatic neuropathy probably resulted from extension of the aortic aneurysm into the iliac vessels overlying the trunks of $\mathrm{L}_{4}-\mathrm{S}_{2}$. Chapman reported three cases of sciatic pain and neuropathy resulting from aneurysms of the common iliac artery or its branches, which directly compressed the underlying nerve trunks. ${ }^{15}$

\section{Diagnosis and management}

Computed tomography is an accurate, noninvasive method of directly imaging masses in the retroperitoneal space. ${ }^{16} \mathrm{~A}$ retroperitoneal haematoma appears on the CT scan as an abnormal soft tissue density which may displace or compress normal structures. Ideally, the scans should be done without contrast enhancement to visualise hyperdensity allowing the CT to distinguish between psoas haematoma and other retroperitoneal lesions.

In patients who develop lumbosacral plexopathy or femoral neuropathy due to iliopsoas haematoma, management is controversial and unresolved. The majority of the described cases are complications of anticoagulant therapy and cessation of therapy is advised. Both immediate surgical decompression of the haematoma and conservative therapy have been recommended with variable results demonstrated for each. ${ }^{16,17}$ Merrick assessed the results of 61 reported cases. ${ }^{17}$ In the 51 patients treated conservatively, $49 \%$ had a complete recovery, $37 \%$ were left with minor impairment and $14 \%$ had little or no improvement. Surgical treatment in ten patients resulted in $50 \%$ total recovery, $40 \%$ minor residual impairment and $10 \%$ without improvement. The decision to intervene to decompress the haematoma and the manner in which the intervention is made is a clinical one, necessitating consideration of the overall condition of the patient.

\section{Summary}

Haemorrhage within the iliopsoas compartment produces femoral nerve dysfunction by compressing the femoral nerve, either within the substance of the iliacus or psoas muscles, in the groove between the iliacus muscle and the psoas muscle or possibly at the level of the inguinal ligament. A large haematoma may extend into and expand the psoas muscle compartment surrounding the femoral nerve and compressing the obturator nerve, and present as a combined femoral-obturator neuropathy. Sciatic neuropathy is related to aneurysmal dilatation or rupture of the common iliacs, resulting in compression of the nerve trunks. Diagnosis of lumbosacral plexopathy is aided by computed tomography. 
Both conservative and surgical management have been applied and, in general, there is no clear basis for favouring one over the other.

\section{References}

1 Wong DHW. Perioperative stroke. Part I: General surgery, carotid artery disease, and carotid endarterectomy.

Can J Anaesth 1991; 38: 347-73.

2 Gottlieb $A$. Aortic reconstructive surgery: anesthetic considerations. Current Opinion in Anaesthesiology 1993: 6: 35-46.

3 Wulf $H$. Epidural anaesthesia and spinal haematoma. Can J Anaesth 1996; 43: 1260-71.

4 Wijdicks EFM. Neurologic complications in critically ill patients. Anesth Analg 1996; 83: 411-9.

5 Veselis $R A$. Ischemic neuropathy presenting as prolonged epidural anesthesia. Reg Anesth 1990; 15: 264-7.

6 Torres GM, Cernigliaro JG, Abbitt PL, et al. Iliopsoas compartment: normal anatomy and pathologic processes. Radiographics 1995; 15: 1285-97.

7 Goodfellow J, Fearn CBA, Matthews JM. Iliacus haematoma. A common complication of haemophilia. J Bone Joint Surg Br 1967; 49: 748-56.

8 Brower TD, Wilde AH. Femoral neuropathy in hemophilia. J Bone Joint Surg Am 1966; 48: 487-92.

9 Kettlekamp DB, Powers SR. Femoral compression neuropathy in the hemorrhagic disorders. Arch Surg 1969; 98: 367-8.

10 Young $M R$, Norris JW. Femoral neuropathy during anticoagulant therapy. Neurology 1976; 26: 1173-5.

11 Boontje $A H$, Haaxma $R$. Femoral neuropathy as a complication of aortic surgery. J Cardiovasc Surg 1987; 28: 286-9.

12 Sasson Z, Mangat I, Peckham KA. Spontaneous iliopsoas hematoma in patients with unstable coronary syndromes receiving intravenous heparin in therapeutic doses. Can J Cardiol 1996; 12: 490-4.

13 Razzuk MA, Linton RR, Darling RC. Femoral neuropathy secondary to ruptured abdominal aortic aneurysms with false aneurysms. J Am Med Assoc 1967; 201: 139-42.

$14 \mathrm{Kubacz}$ GJ. Femoral and sciatic compression neuropathy. Br J Surg 1971; 58: 580-2.

15 Chapman EM, Shaw RS, Kubik CS. Sciatic pain from arteriosclerotic aneurysm of the pelvic arteries. N Eng J Med 1964; 271: 1414-1.

16 Reinstein L, Alevizatos AC, Twardzik FG, DeMarco SJ III. Femoral nerve dysfunction after retroperitoneal hemorrhage: pathophysiology revealed by computed tomography. Arch Phys Med Rehabil 1984; 65: 37-40.

17 Merrick HW, Zeiss J, Woldenberg LS. Percutaneous decompression for femoral neuropathy secondary to heparin-induced retroperitoneal hematoma: case report and review of the literature. Am Surg 1991; 57: 706-11. 